\title{
Harmonic Suppression of Shunt Hybrid Filter using LQR-PSO based
}

\author{
Nor Shahida Hasan ${ }^{1}$, Norzanah Rosmin ${ }^{2}$, Saifulnizam Abd Khalid ${ }^{3}$, Dygku. Asmanissa Awg. Osman ${ }^{4}$, \\ Baharuddin Ishak ${ }^{5}$, Aede Hatib Mustaamal ${ }^{6}$ \\ ${ }^{1,2,4}$ Centre of Electrical Energy System (CEES), POWER Department, Faculty of Electrical Engineering, Universiti \\ Teknologi Malaysia, 81310 Skudai, Johor, Malaysia. \\ ${ }^{3}$ POWER Department, Faculty of Electrical Engineering, Universiti Teknologi Malaysia, 81310 Skudai, Johor, Malaysia. \\ ${ }^{5}$ Tenaga Nasional Berhad (TNB), Protection Unit, Distribution Department, 80100 Jln Yahya Awal, Johor, Malaysia. \\ ${ }^{6}$ Faculty of Education, Universiti Teknologi Malaysia, 81310 Skudai, Johor, Malaysia
}

\section{Article Info}

Article history:

Received Jan 10, 2017

Revised Mar 14, 2017

Accepted Mar 28, 2017

\section{Keyword:}

Active power filter

Harmonic suppression

LQR controller

PSO tuning

Shunt hybrid APF

\begin{abstract}
In linear quadratic regulator (LQR), two different weighting matrices play an important role in presenting the performance of this controller. Instead of using classic common approach, which is trial and error method, this study proposes a particle swarm optimization (PSO) algorithm to track the best solution of the weighting matrices. The proposed algorithm is tested on shunt hybrid active power filter (APF) to mitigate the harmonic contents in voltage and current signals in a nonlinear load system. The modeling work of this proposed system is simulated using MATLAB/Simulink software. From the simulation, the obtained results proved that using PSO in tuning the LQR controller produce smoother nonlinear voltage and current signals. In fact, the amount of current to be injected into network can be reduced up to $95 \%$. Besides, less time is consumed during searching the optimum weighting matrices using the proposed approach.
\end{abstract}

Copyright (c) 2017 Institute of Advanced Engineering and Science. All rights reserved.

Corresponding Author:

Nor Shahida Hasan,

Centre of Electrical Energy System (CEES),

POWER Department,

Faculty of Electrical Engineering,

Universiti Teknologi Malaysia,

81310 Skudai, Johor, Malaysia.

Email: norzanah@utm.my

\section{INTRODUCTION}

The main issue regards to power quality is the power system harmonics. The rapid growth of nonlinear loads and harmonics in current and voltage signals in the power network has become a great deal. Several power quality issues, their causes and other possible solutions in handling these problems were explained in details in [1]. The most conventional methods in dealing with these harmonics problems are using L-C filter and capacitor bank [2], [3]. However, these passive filters have several drawbacks such as fixed compensation, large size, high installation cost and resonance problem [4]. Thus, to overcome these shortcomings, an active power filter (APF) is employed in this work. The ability of APF to inject current into its system promises to provide durability and reliability in its performance.

Technically, APF is designed to produce certain amount of current to be injected into the electrical network in order to drain out the harmonic components of current or voltage signals. The amount of injected current is controlled based on error value between reference and measured current/voltage signals. Later on, the error then converted into pulse width modulation to switch the inverter. In power electronic point of view, this process is quite similar with the voltage/current source inverter [5]. Numbers of publications have proved that shunt APF can successfully mitigate harmonic voltage/current and compensate the reactive power in 
electrical system [6], [7]. Nonetheless, this APF has an adverse trait in its switching process. Its fast switching technique during high currents in power network generates high frequency ripple, which can cause electromagnetic interference in distribution system [5], [6]. In such condition, different types of control algorithms are adopted into APF to improve its steady state and dynamic performance. Various attempts of controllers for APF application has been reviewed and proposed in the previous works, such as $\mathrm{p}-\mathrm{q}$ (the instantaneous reactive-power) theory, dead-beat controller, adaptive controls, Neuro, Fuzzy, wavelet control, sliding mode control, and etc [4]. However, response time of such controllers are limited by microcontroller speed and its performance becomes worst when dealing with nonlinear loads [8]. LQR is a controller that offers optimal tuning of the multivariable feedback gain. LQR is well known of its behavior of robust, efficient and suitable for multi input and multi output [MIMO] systems [9], [10]. Thus a LQR is chosen in this study to mitigate the harmonic voltage and current in shunt hybrid APF.

Theoretically, LQR minimizes quadratic cost function, $J$ that consists of state weighting matrices, Q and control weighting matrices. These two parameters greatly influence the performance of LQR controller. It can be determined using Riccati Equation [11]. Nevertheless, most common approach in obtaining these two parameters is using trial and error method [3], [6]. This classic approach is labor-extensive, time consuming and do not promise the expected performance. Thus, the authors in [12] gave brief explanation on existing optimization techniques to be implemented with LQR, for example, artificial bee colony (ABC), artificial immune systems (AIS), ant colony optimization (ACO), genetic algorithm (GA), artificial neural networks (ANN) and more. However extensive procedure and extra parameters is required to employ these optimization techniques. Therefore, for the sake of simplicity, PSO is proposed in this work to tune the LQR controller in mitigating harmonic voltage and current at nonlinear load.

In comparison with other optimization techniques, PSO promises robust in action, high efficiency, better results, cheaper and less time consuming in tracking the best solution of the fitness [12]. In fact, one of the most attractive feature of PSO is its final solution do not rely on the initial states of the particles. Thus, it makes the coding process in m.file easier [13]. LQR-PSO based had been applied on air craft landing [14], controlling the rotating inverse pendulum [15], stabilizing a two-wheeled wheelchair in balancing mode [16], controlling liquid level in the tank [17] and etc. However, LQR-PSO based implementation on three phase shunt hybrid filter, is somewhat missing in this literature, thus it leads to the main focused of this work.

\section{DESCRIPTION OF THE PROPOSED SYSTEM}

The shunt hybrid model of APF in suppressing harmonic content in electrical system is illustrated in Figure 1. This model comprises of three phase voltage sources, APF, capacitor bank and loads (linear and nonlinear). The phase voltage $\left(u_{s}\right)$ is set as $176.7 \mathrm{~V}_{\text {rms }}$, with resistance $\left(R_{s}\right)$ and inductance $\left(L_{s}\right)$ value of $0.0015 \Omega$ and $0.1 \mathrm{mH}$, respectively. The value of the capacitor $\left(C_{f}\right)$ for the capacitor bank is set to be $99.3 \mu F$ per phase. In this hybrid model, the APF performs the harmonic current mitigation, while the capacitor bank acts as a reactive power compensator besides providing the path to drain the harmonic ripple. The parameter values and the basic structure of this model is based on work in [6], but in this work, some modification in terms of tuning the two weighting matrices using PSO is performed.

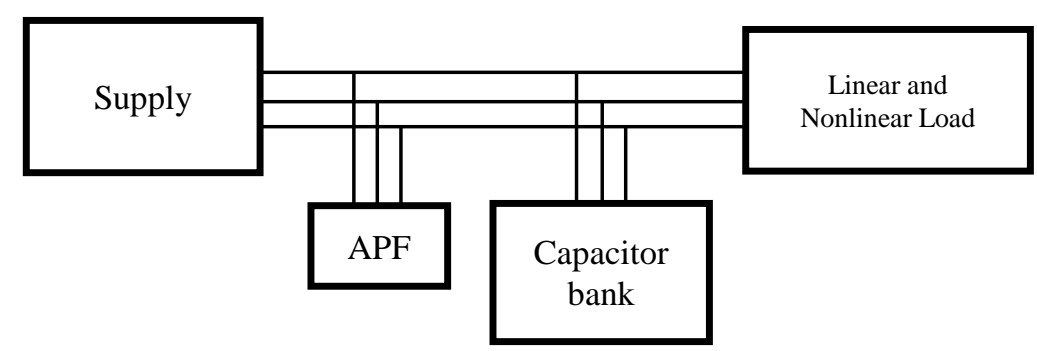

Figure 1. Full system model of shunt hybrid APF

\subsection{Active power filter (APF)}

The APF is constructed using 3-level voltage source inverter (VSI), capacitors $(\mathrm{c} 1=\mathrm{c} 2=500 \mu \mathrm{F})$ and RL filter $\left(R_{f}=0.1 \Omega, L_{f}=2 \mathrm{mH}\right)$. The VSI regenerates the amount of current to be injected into the network. The capacitors of $\mathrm{c} 1$ and $\mathrm{c} 2$ act as energy storage, where they inject certain amount of current into the system every time they detect the error between reference and measured values. This error is then 
converted into pulse width modulation (PWM) signal to switch the IGBT/Diodes. The MATLAB view of the APF model is displayed in Figure 2.

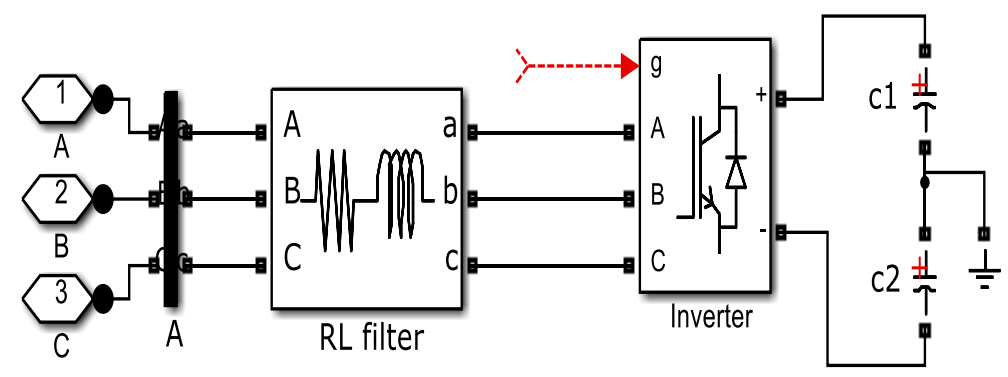

Figure 2. APF model

The reference current of APF is expressed in Equations (1) to (3) [6].

$$
\begin{aligned}
& i_{f a}^{*}(t)=i_{l a}(t)-\frac{2\left(P_{T}+P_{\text {loss }}\right)}{U_{T}} \sin \left(\omega t+\phi_{a 1}\right) \\
& i_{f b}^{*}(t)=i_{l b}(t)-\frac{2\left(P_{T}+P_{\text {loss }}\right)}{U_{T}} \sin \left(\omega t+\phi_{a 1}-2 \pi / 3\right) \\
& i_{f c}^{*}(t)=i_{l c}(t)-\frac{2\left(P_{T}+P_{\text {loss }}\right)}{U_{T}} \sin \left(\omega t+\phi_{a 1}+2 \pi / 3\right)
\end{aligned}
$$

where $i_{f k}^{*}$, is the reference value of filter current for phase $k, i_{l k}$ is the line current of phase $k$ (measured at B_Load block in Figure 1) and subscripts $k$ denotes as phase a, b or c, respectively. $P_{T}$ is the power submitted to load, $U_{t}$ is the sum of peak value of source voltage, $P_{l o s s}$ is the power loss caused by capacitor voltage variation (controlled via a PI controller), $\phi_{a 1}$ is the phase angle of the fundamental component of load voltage at phase $a$ and $\omega$ is the angular speed at frequency of $60 \mathrm{~Hz}$ [6].

\subsection{Shunt capacitor bank}

In this shunt hybrid APF, the capacitor bank (CB) acts as a passive power filter, in which helps to provide the draining path of the harmonic currents. The feature advantages of $\mathrm{CB}$ are low in cost and flexibility in its installation [18].

\subsection{Nonlinear load}

Nonlinear load system that shown in Figure 1 is composed with linear and nonlinear loads. The nonlinear load is 6-pulse diode rectifier while the linear load is a 3-phase series resistance-inductance branch per phase $(R=15.4 \Omega$ and $L=15.4 \mathrm{mH})$. The linear load is shunt connected to nonlinear load.

\section{LQR CONTROLLER: PSO TUNING BASED}

In this study, the performance index of LQR is tuned using PSO. PSO is chosen due to its simplicity whereby only certain parameters are needed in writing the optimization code. Thus, enable faster tracking process in searching the best value of corresponding dimensions.

\subsection{Linear quadratic regulator $(\mathbf{L Q R})$}

Based on state equation of $x=A x+B u$ and $y=C x+D u$, the equivalent state space equation, $x$ and the state vector $x$ for Figure 1 is presented as written in Equation (4) and Equation (5) accordingly [6]. 


$$
\begin{aligned}
& \bar{x}=\left[\begin{array}{cccc}
-\frac{R_{s}}{L_{s}} & 0 & 0 & -\frac{1}{L_{s}} \\
0 & -\frac{R_{f}}{L_{f}} & 0 & -\frac{1}{L_{f}} \\
0 & 0 & -\frac{R_{e q}}{L_{e q}} & \frac{1}{L_{e q}} \\
\frac{1}{C_{f}} & \frac{1}{C_{f}} & -\frac{1}{C_{f}} & 0
\end{array}\right] x+\left[\begin{array}{c}
\frac{1}{L s} \\
0 \\
0 \\
0
\end{array}\right] u_{s}+\left[\begin{array}{c}
0 \\
\frac{V_{d c}}{L_{f}} \\
0 \\
0
\end{array}\right] m \\
& x=\left[\begin{array}{llll}
i_{f} & i_{c} & i_{l} & u_{c}
\end{array}\right] T
\end{aligned}
$$

where $V_{d c}$ is the rated value of DC voltage, $i_{f}$ is the filter current, $i_{l}$ is the load current, $i_{c}$ and $u_{c}$ is the current and voltage of capacitor bank, respectively. $R_{e q}$ and $L_{e q}$ is the equivalent resistance and equivalent inductance for linear and nonlinear load. $m$ is the linear state-feedback law, $(m=-\mathbf{K} x)$ to minimize the quadratic cost function, $J$ as written in Equation (6) [6]. $\mathbf{K}$ is the gain matrix, which can be obtained from Riccati Equation [11].

$$
J=\frac{1}{2} \int_{0}^{\infty}\left(x^{T} Q x+u^{T} R u\right) d t
$$

where $Q$ and $R$ are the semi-definite and a positive real symmetric weight matrix of state matrix $x(t)$ and input matrix $u(t)$. The important matter in LQR control is obtaining the value of Qs and $\mathrm{R}$ weighting matrices. It becomes more difficult when the system has more parameters and more input and output variables. Thus, to overcome these restraints and reducing time-consuming approach, a PSO is adopted into the system to tune the value of $\mathrm{Q}$ and $\mathrm{R}$ weighting matrices in an easier and faster way. $\mathrm{X}$

\subsection{Particle swarm optimization (PSO)}

PSO algorithm is developed based on the nature behavior of swarm, fish flocking, bees, whales or other animals while they are looking for their food [19]. Technically, the involved particles will fly around with the updated velocity (Equation (7)) towards a new position (Equation (8)). They will keep on competitively flying until they find the best value for the fitness function [20].

$$
\begin{aligned}
& v_{i}(t)=v_{i}(t-1)+c_{1} w_{1}\left(p_{\text {best }}-x_{i}(t-1)\right)+c_{2} w_{2}\left(g_{\text {best }}-x_{i}(t-1)\right) \\
& x_{i}(t)=x_{i}(t-1)+v_{i}(t)
\end{aligned}
$$

In this work, the fitness value is the integrated absolute error (IAE) between the references value of state vector and its measured state vector $x$. Since the state vector consists of filter current, voltage and capacitor current, thus, all the obtained errors between these values will be summed up and stored in the workspace as IAE. The reference value for filter current was given in section 2.1. While the reference value for current, $i_{c, k}^{*}$ and voltage, $v_{c, k}^{*}$ of capacitor bank is obtained from positive sequence extraction of current and voltage source [13]. On the other hand, the $4 \times 4$ diagonal matrixes of $\mathrm{Q}$ and $1 \mathrm{x} 1$ matrix of $\mathrm{R}$ will be set as the dimension. Therefore, the dimension is set to 5 in the Matlab coding. Dimension can be defined as the parameters to be optimized. The pseudo code for the PSO-based LQR is as follows [13]:

Initialize each particle.

Do

Find R, Q,P (using Reccati Equation) and $\mathbf{K}$ using $m=-\mathbf{K} x$

Calculate $x(t)$

Call the simulink file

Calculate the IAE

Find $p_{\text {best }}$, if the present $\operatorname{IAE}(\mathrm{t})<\operatorname{IAE}\left(p_{\text {best }}\right)$ then $x(t)$ is the current $p_{\text {best }}$ 
Set the current value as the new $p_{\text {best }}$

End

Choose the particle with the least IAE value of all the particles as the $g_{\text {best }}$.

For each Particle:

End

Calculate particles velocity, update particles position and get $\mathrm{Q}$ and $\mathrm{R}$.

Until all stopping criteria is met .

The parameters used during initialization and the whole processes are listed in Table 1 below. The upper and lower level value is obtained from publication in [6].

Table 1. Parameters value for PSO

\begin{tabular}{cc}
\hline Parameters & Value \\
\hline Number of particles & 20 \\
Number of iterations & 10 \\
Number of dimension & 5 \\
$w_{1}, w_{2}, c_{1}, c_{2}$ & $0.4,0.9,1.5,1.5$ \\
Upper band & {$[30,30,30,0,0.1]$} \\
Lower band & {$[0.0001,0.0001,0.0001,0.0001,0.0001]$} \\
\hline
\end{tabular}

After 10 iterations with 20 particles involved, the weighting matrices of Q and R, and feedback gain matrix for each phase, $\mathbf{K}_{k}$, are obtained. The results for the gains of $\mathbf{K}$ are given as written in Equation (9) to Equation (11).

$$
\begin{aligned}
& Q=\operatorname{diag}\left(\left[\begin{array}{l}
5.72677 .543521 .6203 \\
0.0001])
\end{array}\right.\right. \\
& R=0.0625 \\
& \mathbf{K}_{a}=\left[\begin{array}{lll}
9.5373 & 5.1246 & 20.9674
\end{array}\right] \\
& \mathbf{K}_{b}=\left[\begin{array}{lll}
9.5373 & 5.1247 & 20.9625
\end{array}\right] \\
& \mathbf{K}_{c}=\left[\begin{array}{lll}
9.5373 & 5.1247 & 20.9625
\end{array}\right]
\end{aligned}
$$

These Qs, R and Ks are affected by the value of $R_{e q}$ and $L_{e q}$. Since the involved nonlinear and linear loads are in balanced condition, thus, the obtained linear feedback gain, $K_{k}$ is almost similar for each phase. These values will keep on changing every time the simulation is run due to the existing code of 'rand()' in parameter Q and R. However, this value will not give much effect on the simulation results as long as the simulation is run between the determined upper and lower band of PSO.

\subsection{Pulse width modulation (PWM) analysis}

Since the feedback gain, $\mathbf{K}$ value is measured for each phase, thus, in order to generate a switching signal for phase $a$, all the measured IAE of the filter current, capacitor bank current and capacitor bank voltage are summed up before entering the hysteresis band. Later on, these signals will be converted into gating signal to switch the three levels IGBT/Diodes. The MATLAB view of these steps is shown in Figure 3. For the switching signal generation for phase $b$ and $c$, same step/process is applied. 


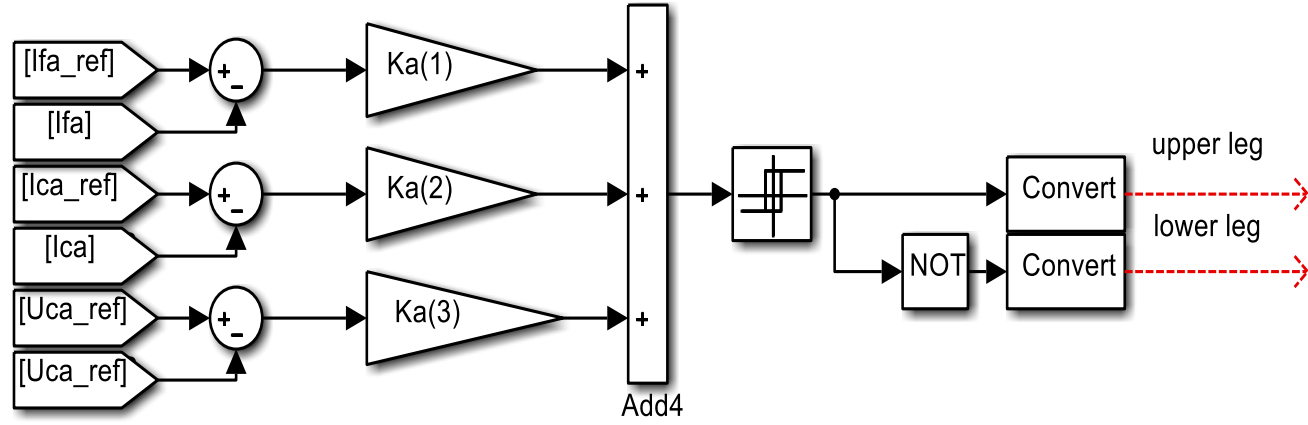

Figure 3. Switching signals of IGBT/Diodes

\section{RESULTS AND DISCUSSION}

Figure 4 shows the source voltage, source current, load voltage and load current signals when the shunt Hybrid APF system is self-controlled. Meanwhile, Figure 5 shows the same signals but with the existence of LQR-PSO based control in the proposed APF system. From Figure 4(a) and Figure 4(b), it can be observed that, without the implementation of LQR based PSO, the current and voltage signals at the source side are influenced by the harmonic factor. Similar situation can be depicted to the generated voltage and current signals at the load side, as depicted in Figure 4(c) and Figure 4(d), respectively. This is because, at $0.04 \mathrm{sec}$, APF starts to inject the filter current into the network. However, due to the fast switching behavior of APF [5], high frequency ripple is generated at voltage and current signals on both sides. Thus, this proved that in the absence of an appropriate controller, the APF could increase the harmonic contents in the current and voltage signals.

Meanwhile, as can be seen in Figure 5(a-d), the generated signals for source voltage, source current, load voltage and load current are smoother and free from high frequency ripple compared to the signals in Figure 4(a-d). The obtained voltage signal at load side (as shown in Figure 5(c)) is 150V, which is equaled to the voltage at supply side (as shown in Figure 5(a)). This is because the balanced non linear load is connected in parallel with supply. While, the current values on the supply side remains constant at $12 \mathrm{~A}$ once the hysteresis controller is switched on. On the contrary, load current remains constant at approximately 11A throughout the simulation times. The reduction of current values from supply side (12A) to the load side $(11 \mathrm{~A})$ is due to the power losses in the system network during power transmission.
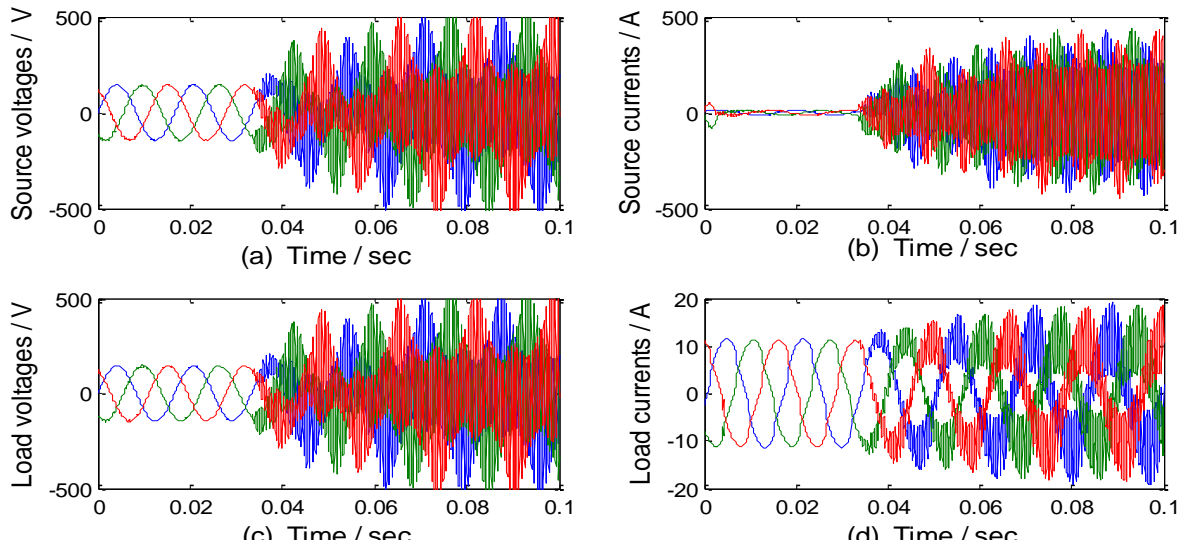

(c) Time $/ \mathrm{sec}$

(d) Time / sec

Figure 4. Results of shunt hybrid filter without the linear feedback gain of LQR: (a) Voltage source, (b) Current source, (c) Load voltage, (d) Load current

Obviously, with the presence of LQR-PSO based controller, with the tuned value of feedback gain, $\mathbf{K}$, the harmonic content in both source and load sides are suppressed, as shown in Figure 5(a) to Figure 5(d). Thus, with the existence of tuned K parameter in the LQR-PSO based controller, smoother voltage and current signals can be supplied to the load side. However, as can be seen in this figure, there are 
some fluctuations shown in the early simulation stage, as can be observed in Figure 5(b), e.g. time between 0 to 0.02 second. This is caused by the setting time of the hysteresis band. Nevertheless, once the hysteresis band is at on state, the source signals become smoother.

Figure 6 shows the current injected from shunt Hybrid APF at phase a. This injected current is based on the summation of the nonlinear load and linear load. Since the applied linear and nonlinear load applied in each phase is balanced, thus the amount of injected currents for phase $a$ are almost the same for phase $b$ and phase $c$. From this current signal, it can be observed clearly that with the existence of LQR-PSO based controller, the injected current from APF into the power network is reduced. Without the LQR-PSO based, almost $\pm 60 \mathrm{~A}$ is required to be injected into the power network to serve the load. Conversely, with the implementation of LQR-PSO based on shunt hybrid APF, only approximately $\pm 3 \mathrm{~A}$ is required to be injected into such power network. Besides, from the simulation work that has been done, the advantages of PSO are proven. From the tuning activities, it was found that PSO offers fast tuning process, fast convergence and less computational work.
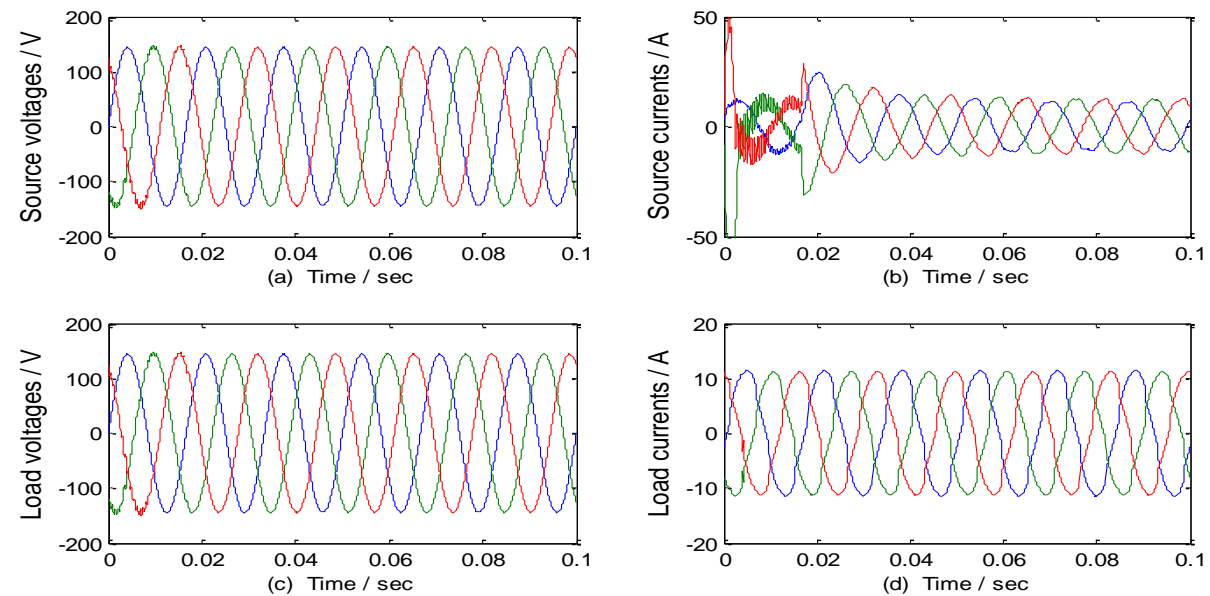

Figure 5. Results of shunt hybrid filter with the linear feedback gain of LQR: (a) Voltage source, (b) Current source, (c) Load voltage, (d) Load current

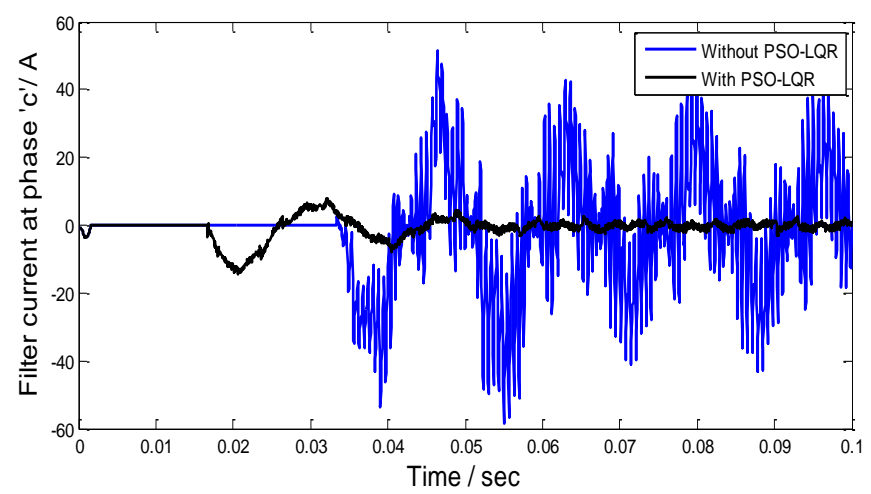

Figure 6. Filter current of APF to be injected into the network

\section{CONCLUSION}

In this study, a shunt hybrid APF model with the presence of LQR controller was successfully designed and constructed using MATLAB/Simulink software environment. The LQR controller has been placed in the APF model to mitigate the current and voltage harmonics in a network with nonlinear load. To obtain the appropriate weighting matrices of the LQR controller, PSO algorithm was used. From the obtained simulation results, it had been shown that the harmonic contents in the voltage and current signals of the nonlinear load can be suppressed successfully when LQR-PSO based controller was built in the APF. The injected current from APF was also reduced up to $95 \%$, therefore improving the APF behavior in terms of its 
fast switching technique. The application of PSO technique in tracking the best solution of Q and R weighting matrices has reduced the weakness of trial and error method. As conclusion, using PSO in LQR approach is more convenient, presents more powerful in computational technique and less time consuming than the conventional technique.

\section{ACKNOWLEDGEMENTS}

The authors would like to thank RMC, FKE and CEES, UniversitiTeknologi Malaysia and the Ministry of Higher Education of Malaysia for the financial support provided under RUG(12H00) and FRGS(4F794) to carry out this research.

\section{REFERENCES}

[1] M. Gao and K. Awodele, "Investigation of power electronics solutions to power quality problems in distribution networks," in AFRICON, Addis Ababa, pp. 1-6, 2015.

[2] T. Hao, et al., "Linear quadratic optimal control of a single-phase grid-connected inverter with an LCL filter," in Industrial Electronics (ISIE), 2012. IEEE International Symposium on, pp. 372-376, 2012.

[3] A. Kaszewski, et al., "The LQ controller for the 3-phase 4-leg inverter with an LC output filter- Choosing the right reference frame," in Power Electronics and Applications (EPE), 2013 15th European Conference on, pp. 1-9, 2013.

[4] R. Zahira and A. P. Fathima, "A Technical Survey on Control Strategies of Active Filter for Harmonic Suppression," Procedia Engineering, vol. 30, pp. 686-693, 2012.

[5] Z. Salam, et al., "Harmonics mitigation using active power filter: a technological review," Elektrika, vol. 8, pp. 1726, 2006.

[6] H. A. R. Carranza, et al., "Suppression of parallel resonance and mitigation of harmonic distortion through shunt active power compensation," International Journal of Electrical Power \& Energy Systems, vol. 75, pp. 152-161, 2016.

[7] R. Belaidi, et al., "Improvement of the electrical energy quality using a Shunt Active Filter supplied by a photovoltaic generator," Energy Procedia, vol. 6, pp. 522-530, 2011.

[8] H. Komurcugil, et al., "Optimal control for single-phase UPS inverters based on linear quadratic regulator approach," in International Symposium on Power Electronics, Electrical Drives, Automation and Motion, 2006. SPEEDAM 2006., Taormina, pp. 1137-1142, 2006.

[9] B. Kedjar and K. Al-Haddad, "LQR with Integral Action for Phase Current Control of Constant Switching Frequency Vienna Rectifier," in 2006 IEEE International Symposium on Industrial Electronics, Montreal, Que., pp. 1461-1466, 2006

[10] S. Mobayen, et al., "Linear quadratic optimal control system design using particle swarm optimization algorithm," International Journal of the Physical Sciences, vol. 30, pp. 6958 - 6966, 2011.

[11] T. Nguyen and Z. Gajic, "Solving the Matrix Differential Riccati Equation: A Lyapunov Equation Approach," IEEE Transactions on Automatic Control, vol. 55, pp. 191-194, 2010

[12] K. Hassani and W. S. Lee, "Optimal Tuning of Linear Quadratic Regulators Using Quantum Particle Swarm Optimization," presented at the Proceedings of the Int. Conference of Control, Dynamic Systems, and Robotics, Ottawa, Ontario, Canada, 2014.

[13] M. I. Solihin, et al., "Comparison of LQR and PSO-based state feedback controller for tracking control of a flexible link manipulator," in Information Management and Engineering (ICIME), 2010 The 2nd IEEE International Conference on, pp. 354-358, 2010.

[14] J. Hamidi, "Control System Design Using Particle Swarm Optimization (PSO)," International Journal of Soft Computing and Engineering (IJSCE), vol. 1, pp. 116-119, 2012.

[15] Y. K. Wei, et al., "LQ Regulator Design Based on Particle Swarm Optimization," presented at the 2006 IEEE International Conference on Systems, Man, and Cybernetics, Taipei, Taiwan, pp. 4142-4145, 2006.

[16] A. Aula, et al., "PSO-based State Feedback Regulator for Stabilizing a Two-wheeled Wheelchair in Balancing Mode," presented at the Control Conference (ASCC), 2015 10th Asian, Kota Kinabalu, 2015.

[17] N. A. Selamat, et al., "Comparison of LQR and PID controller tuning using PSO for Coupled Tank System," in Signal Processing \& Its Applications (CSPA), 2015 IEEE 11th International Colloquium on, Kuala Lumpur, pp. 46-51, 2015.

[18] C. S. Lam and C. W. Wong, "Design and Control of Hybrid Active Power Filters," Springer Heidelberg New York Dordrecht London: SpringerBriefs in Electrical and Computer Engineering, 2014.

[19] Q. Bai, "Analysis of Particle Swarm Optimization Algorithm," Computer and Information Science, vol. 3, pp. 180$184,2010$.

[20] K. W. Yu and Z. L. Huang, "LQ Regulator Design Based on Particle Swarm Optimization," in 2006 IEEE International Conference on Systems, Man and Cybernetics, Taipei, pp. 4142-4145, 2006. 